\title{
Design of nonlinear GaAs/AlGaAs second harmonic converters
}

\author{
Alessandro Massaro*, Vittorianna Tasco, Maria Teresa Todaro, \\ Roberto Cingolani, Massimo De Vittorio, Adriana Passaseo ${ }^{1}$ \\ National Nanotechnology Laboratory of CNR-INFM, Distretto Tecnologico-ISUFI, Università del Salento, Via Arnesano, 73100 Lecce, \\ Italy
}

\section{A R T I C L E I N F O}

\section{Article history:}

Available online 18 July 2009

\section{Keywords:}

Second harmonic converter

Nonlinear waveguides

\begin{abstract}
A B S T R A C T
In this work we present the design of a three-dimensional wavelength converter consisting of GaAs/AlGaAs nonlinear ridge waveguide with a quasi-phase-matched grating. A fundamental mode at $\lambda_{\mathrm{FU}}=1.55 \mu \mathrm{m}$, and a copropagating second harmonic mode at $\lambda_{\mathrm{SH}}=0.775 \mu \mathrm{m}$ are considered. The second harmonic generation process in the $\chi^{(2)}$ nonlinear waveguides is analysed by analytical and numerical approaches. The numerical approach is performed by the Hertzian potential formulation typically used for the modelling of $\chi^{(2)}$ nonlinear processes. A good agreement between analytical and numerical results is observed.
\end{abstract}

(c) 2009 Elsevier Ltd. All rights reserved.

\section{Introduction}

Wavelength conversions of lasers are fundamental techniques in optoelectronics. These techniques require the phase coincidence of optical waves generated in nonlinear medium to obtain high conversion efficiency.

Previous works have analyzed complex and rigorous techniques in order to achieve a high second harmonic (SH) generation efficiency in $\mathrm{GaAs} / \mathrm{AlGaAs}$ and $\mathrm{LiNbO}_{3}$ waveguides including quantumwell domain-distorted quasi-phase-matched waveguides [1], orientation patterned structures [2], and pump detuning technique [3]. The goal of the presented work is to provide design criteria for a high SH efficiency in a passive phase matched waveguides which can be obtained by a single patterning/etching process by overcoming all the problems associated to complex epitaxial technologies or complex regrowth processes.

\footnotetext{
* Corresponding author. Tel.: +39 0832 298373; fax: +39 0832298386.

E-mail address: alessandro.massaro@unile.it (A. Massaro).

1 Permanent address: IMM-CNR sezione Lecce, University Campus, Lecce-Monteroni 73100, Italy.
} 

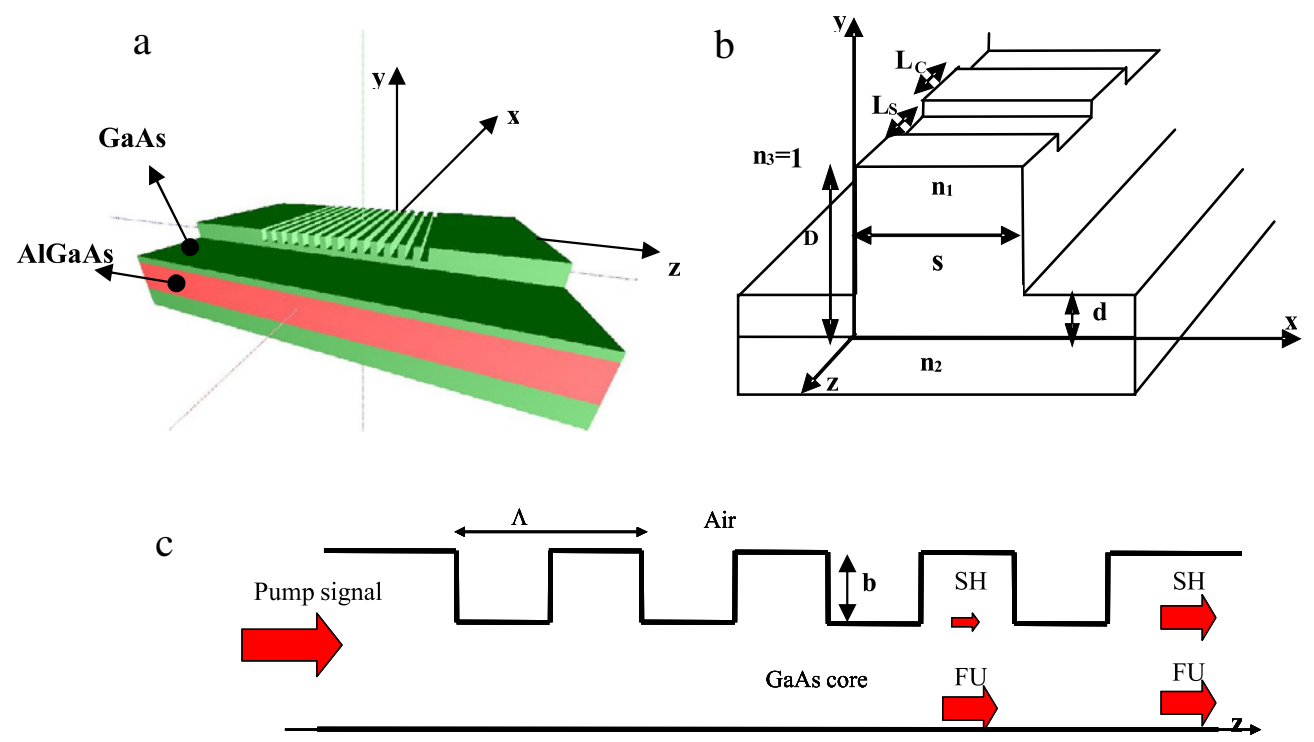

Substrate

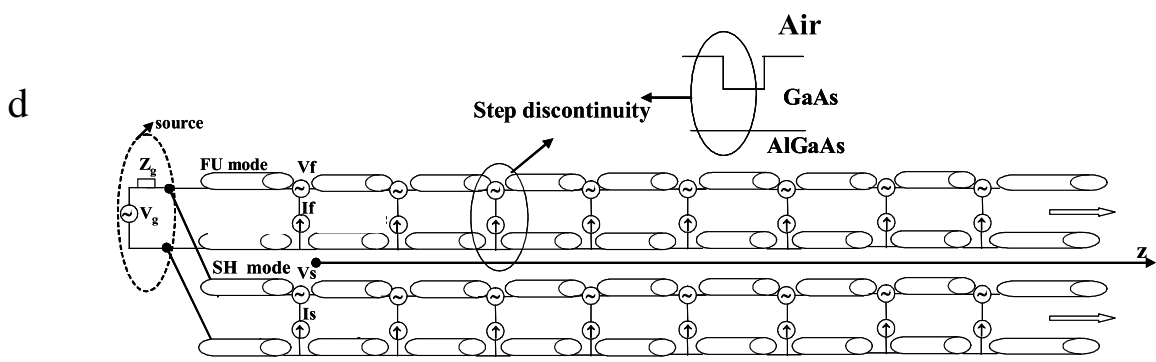

Fig. 1. (a) 3D ridge waveguide. (b) Geometrical and optical parameters of the 3D ridge waveguide. (c) Schematic diagram of the $\chi^{(2)}$ nonlinear process inside an asymmetrical slab waveguide with GaAs nonlinear core: the fundamental (FU) and second harmonic (SH) modes are copropagating modes. (d) Transmission line model of the discontinuous ridge waveguide.

Among various kinds of phase matching devices, quasi-phase matching (QPM) [4-6] is a most promising method because QPM can use the maximum nonlinear optical tensor component with flexibility of designed phase matching wavelength. The QPM application in high efficiency second harmonic $(\mathrm{SH})$ processes is analyzed in this work in which we model the $\chi^{(2)}$ nonlinear process for the GaAs/AlGaAs ridge waveguide, showed in Fig. 1(a) and (b) for different angles of view, with a quasi-phase-matched grating showed in the longitudinal section of Fig. 1(c). The ridge waveguide allows to focus in the ridge region the fundamental mode power generated by a pump source at the telecommunication wavelength of $\lambda_{0}=1.55 \mu \mathrm{m}$. Through the $\chi^{(2)}$ nonlinear process the fundamental mode $\left(\lambda_{\mathrm{FU}}=1.55 \mu \mathrm{m}\right)$ generates, in the ridge converter, a copropagating second harmonic $(\mathrm{SH})$ signal $\left(\lambda_{\mathrm{SH}}=0.775 \mu \mathrm{m}\right)$ which is enhanced by means of the quasiphase-matched grating. The efficient ridge waveguide is designed and optimized by analytical and numerical approaches. The used numerical approach, validated by comparison with experimental results of nonlinear GaAs/AlGaAs waveguides found in literature [4,7], includes the losses due to the backscattering generated by the grating. It is based on nonlinear wave equations associated to the circuital approach which considers the time-domain wave propagating in nonlinear transmission lines. The transmission lines represent the propagating modes of a nonlinear optical waveguide. Each 


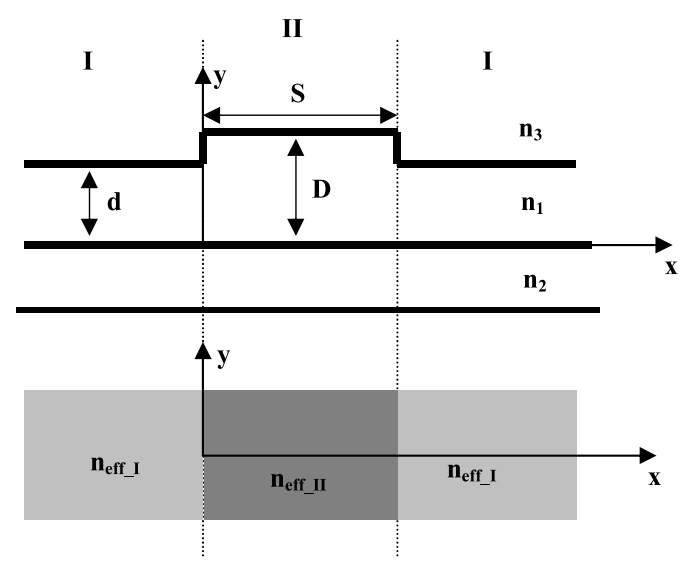

Fig. 2. Geometrical parameters of EDC analysis.

propagating mode is a solution of the scalar Helmholtz wave equation [8-10] and is associated to a transmission line. This analogy allows to model a nonlinear optical waveguide as a set of transmission line circuits taking into account the dielectric interfaces along the propagating direction as voltage and current generators (see Fig. 1(d)) [7,11] which model accurately the discontinuous regions of the periodic nonlinear waveguide. The generators are placed directly on the dielectric interfaces by optimizing the numerical error of the temporal second derivates of the Helmholtz equation at the dielectric boundary condition, as proved through the comparison experimental results for a fundamental wavelength of $\lambda_{\mathrm{FU}}=1.955 \mu \mathrm{m}$ [7]. By using this approach the second harmonic generation process is treated as a simple equivalent scalar problem with a numerical accuracy provided by the generators. In the three-dimensional (3D) case the proposed algorithm solves only two scalar equations instead of six, and subsequently all the electromagnetic (EM) field components are obtained by the two scalar potentials as in the Hertzian potential formulation (HPF) [7,11]. We design and optimize the ridge waveguide converter with grating by considering the transmission line model of Fig. 1(d), and the analytical approaches such as effective dielectric constant (EDC) method [12] and coupled mode theory [5,6]. The numerical and the analytical approaches include the analysis of the losses due to the grating backscattering.

\section{Analytical modeling}

By assuming a transverse electric (TE) polarized source field we focus the analysis on the TE ridge modes. In particular the ridge waveguide is characterized by [12] TE with respect to the $y$-axis (TE with $E_{y}=0$ ) modes. The EDC method allows to define these modes. As shown in Fig. 2 we study regions I and II separately. First we evaluate the propagation constants in the $y$-direction then the effective constants $n_{\text {eff_I }}$ and $n_{\text {eff_II }}$ (belonging to regions I and II, respectively), and then the propagation constants along the $x$-direction. The $T E^{y}$ modes related to the central guiding region II are defined in Table 1 , where $\beta, k_{y}, k_{x}$ and $\varphi_{y}$ are the propagation constant along the $z$-direction, the propagation constant along the $y$-direction, the propagation constant along the $x$-direction, and the phase shift [12], respectively. The $T E^{y}$ modes are referred to the fundamental and $\mathrm{SH}$ copropagating modes. With the introduction of the grating with period $\Lambda$, the conversion efficiency of these modes is calculated by the coupled mode theory. In particular by indicating with $A^{\omega}$ the amplitude of the pump fundamental field and with $A^{2 \omega}$ the amplitude of the SH wave we obtain $[5,6]$

$$
\left\{\begin{array}{l}
\frac{\mathrm{d} A^{\omega}(z)}{\mathrm{d} z}+j\left(2 k_{L}^{\omega} \cos \frac{2 \pi}{\Lambda} z\right) A^{\omega}(z)=-j\left[k_{N L} \exp (j 2 \delta z)\right]^{*}\left[A^{\omega}(z)\right]^{*} A^{2 \omega}(z) \\
\frac{\mathrm{d} A^{2 \omega}(z)}{\mathrm{d} z}+j\left(2 k_{L}^{2 \omega} \cos \frac{2 \pi}{\Lambda} z\right) A^{\omega}(z)=-j k_{N L} \exp (j 2 \delta z)\left[A^{\omega}(z)\right]^{2}
\end{array}\right.
$$


Table 1

$T E^{y}$ modes in the guiding region.

\begin{tabular}{|c|c|}
\hline & $T E^{y}$ \\
\hline$E_{x}$ & $\omega^{\mathrm{FU}, \mathrm{SH}} \mu_{0} \beta^{\mathrm{FU}, \mathrm{SH}} \frac{\cos \left(k_{x}^{\mathrm{FU}, S \mathrm{SH}} x\right)}{\cos \left(k_{x}^{\mathrm{FU}, \mathrm{SH}} S\right)} \cdot \frac{\cos \left(k_{y, I I}^{\mathrm{FU}, S \mathrm{H}} y-\varphi_{y, I I}^{\mathrm{FU}, \mathrm{SH}}\right)}{\cos \left(k_{y_{-} I I}^{\mathrm{FU}, \mathrm{SH}} D-\varphi_{y \_}^{\mathrm{FU}, \mathrm{SH}}\right)}$ \\
\hline$E_{y}$ & 0 \\
\hline$E_{z}$ & $j \omega^{\mathrm{FU}, S \mathrm{SH}} \mu_{0} k_{x}^{\mathrm{FU}, \mathrm{SH}} \frac{\sin \left(k_{x}^{\mathrm{FU}, S \mathrm{SH}} x\right)}{\cos \left(k_{x}^{\mathrm{FU}, \mathrm{SH}} S\right)} \cdot \frac{\cos \left(k_{y \unlhd I}^{\mathrm{FU}, S \mathrm{SH}} y-\varphi_{y, I I}^{\mathrm{FU}, \mathrm{SH}}\right)}{\cos \left(k_{y \_}^{\mathrm{FU}, \mathrm{SH}} D-\varphi_{y \_}^{\mathrm{FU}, S \mathrm{SH}}\right)}$ \\
\hline$H_{x}$ & 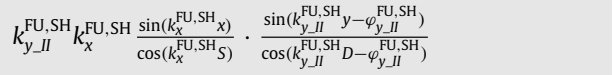 \\
\hline$H_{y}$ & 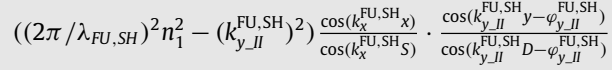 \\
\hline$H_{z}$ & 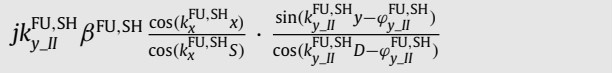 \\
\hline
\end{tabular}

with

$$
\begin{aligned}
& k_{L}^{\omega}=\frac{\omega \varepsilon_{0}}{4} \int\left[E_{x}^{\omega}(x)\right]^{*} \Delta \varepsilon E_{x}^{\omega}(x) \mathrm{d} x \\
& k_{L}^{2 \omega}=\frac{2 \omega \varepsilon_{0}}{4} \int\left[E_{x}^{2 \omega}(x)\right]^{*} \Delta \varepsilon E_{x}^{2 \omega}(x) \mathrm{d} x \\
& k_{N L}^{=} \frac{2 \omega \varepsilon_{0}}{4} \int\left[E_{x}^{2 \omega}(x)\right]^{*} \tilde{\chi}_{N L}\left[E_{x}^{\omega}(x)\right]^{2} \mathrm{~d} x
\end{aligned}
$$

where

$$
2 \delta=\beta^{2 \omega}-\left(2 \beta^{\omega}+2 \pi / \Lambda\right)
$$

is the phase mismatch between the fundamental and SH fields,

$$
\Delta \varepsilon=\left(\varepsilon_{1}-\varepsilon_{2}\right) \frac{\sin (a \pi)}{\pi} \exp [-j z 2 \pi / \Lambda]
$$

is the first term of the permittivity Fourier series, and finally

$$
\tilde{\chi}_{N L}=\left(\chi_{1}^{(2)}-\chi_{2}^{(2)}\right) \frac{\sin (a \pi)}{\pi} \exp [-j z 2 \pi / \Lambda]
$$

is the first term of the nonlinear coefficient Fourier series. The parameter $a$ denotes the duty ratio of the period, and the term $\left(\chi_{1}^{(2)}-\chi_{2}^{(2)}\right)$ indicates the periodically switched nonlinearity related to the GaAs/AlGaAs ridge waveguide. The losses due to the wave reflection at each step discontinuity are considered through the canonical form of the transmission matrix which takes into account the following relationship between the currents and voltages at the input and the output of the analyzed waveguide [12]

$$
\left(\begin{array}{c}
V_{\text {input }}^{\omega, 2 \omega} \\
I_{\text {input }}^{\omega, 2 \omega}
\end{array}\right)=\mathbf{T}\left(\begin{array}{c}
V_{\text {output }}^{\omega, 2 \omega} \\
I_{\text {output }}^{\omega, 2 \omega}
\end{array}\right)
$$

where $\mathbf{T}$ is the total transmission matrix obtained by the following canonical decomposition $\mathbf{T}=$ $\mathbf{M S M}^{-1}(\mathbf{S}$ is the diagonal matrix whose elements are the eigenvalues of $\mathbf{T}), V^{\omega, 2 \omega}=A^{\omega, 2 \omega}=E_{x}^{\omega, 2 \omega}$, and $I^{\omega, 2 \omega}=H_{y}^{\omega, 2 \omega}$.

\section{Numerical Hertzian potential modeling}

In order to design the GaAs/AlGaAs waveguide of Fig. 1 we consider the Hertzian potential formulation used to solve $\chi^{(2)}$ nonlinear problems in discontinuous optical waveguides [7]. The $\mathrm{HPF}$ is based on nonlinear wave equations associated to the circuital approach which considers 
the time-domain wave propagating in nonlinear transmission lines reported in Fig. 1(d) [7-11]. The transmission lines represent the two propagating modes of the nonlinear optical waveguide (fundamental and SH field). Each propagating mode is solution of the scalar Helmholtz wave equation and is associated to a transmission line characterized by the modal effective refractive index which change with the thickness along the waveguide. This analogy allows to model a nonlinear optical waveguide as a set of two transmission line circuits which take into account the dielectric interfaces along the propagating direction as voltage and current generators $V_{f}, I_{f}, V_{s}$, and $I_{s}$, as reported in Fig. 1(d) [7,11]. The generators, placed directly at the interface nodes, allow to optimize the numerical error at the dielectric boundaries. By using this approach the SH generation process is treated as a simple equivalent scalar problem. The coupled equations numerically solved are the following Helmholtz scalar equations

$$
\begin{aligned}
& \nabla^{2} \Psi^{\mathrm{FU}}=\mu_{0} \varepsilon_{0}\left(N_{i}^{\omega}\right)^{2} \frac{\partial^{2} \Psi^{\mathrm{FU}}}{\partial t^{2}}+2 \mu_{0} \varepsilon_{0} \chi^{(2)}\left(\Psi^{\mathrm{FU}} \frac{\partial^{2} \Psi^{\mathrm{SH}}}{\partial t^{2}}+\Psi^{\mathrm{SH}} \frac{\partial^{2} \Psi^{\mathrm{FU}}}{\partial t^{2}}+2 \frac{\partial \Psi^{\mathrm{FU}}}{\partial t} \frac{\partial \Psi^{\mathrm{SH}}}{\partial t}\right) \\
& \nabla^{2} \Psi^{\mathrm{SH}}=\mu_{0} \varepsilon_{0}\left(N_{i}^{2 \omega}\right)^{2} \frac{\partial^{2} \Psi^{\mathrm{SH}}}{\partial t^{2}}+2 \mu_{0} \varepsilon_{0} \chi^{(2)}\left(\Psi^{\mathrm{FU}} \frac{\partial^{2} \Psi^{\mathrm{FU}}}{\partial t^{2}}+\frac{\partial \Psi^{\mathrm{FU}}}{\partial t} \frac{\partial \Psi^{\mathrm{FU}}}{\partial t}\right)
\end{aligned}
$$

where $\psi^{\mathrm{FU}}$ and $\psi^{\mathrm{SH}}$ indicate the fundamental and the second harmonic field respectively, and, $N_{i}^{\omega, 2 \omega}$ represents the $T E^{y}$ effective refractive index evaluated along the waveguide. By the finite difference discretization [13], Eqs. (9) and (10) become

$$
\begin{aligned}
& \Psi_{\mathrm{FU}}^{n+1}(i) \cdot\left(\mu_{0} \varepsilon_{0}\left(N^{\omega}\right)^{2} \Delta^{2} z+2 \mu_{0} \varepsilon_{0} \chi^{(2)} \Delta^{2} z \Psi_{\mathrm{SH}}^{n}(i)\right) \\
& \quad=\Psi_{\mathrm{FU}}^{n}(i) \cdot\left[\left(-2 \Delta^{2} t+2 \mu_{0} \varepsilon_{0}\left(N^{\omega}\right)^{2} \Delta^{2} z-2 \mu_{0} \varepsilon_{0} \chi^{(2)} \Delta^{2} z \cdot\left(\Psi_{\mathrm{SH}}^{n+1}(i)-2 \Psi_{\mathrm{SH}}^{n}(i)+\Psi_{\mathrm{SH}}^{n-1}(i)\right)\right.\right. \\
& \left.\quad-2 \mu_{0} \varepsilon_{0} \chi^{(2)} \Delta^{2} z \Psi_{\mathrm{SH}}^{n}(i)\right]+4 \mu_{0} \varepsilon_{0} \chi^{(2)} \Delta^{2} z \Psi_{\mathrm{FU}}^{n+1 / 2}(i) \cdot\left(-\Psi_{\mathrm{SH}}^{n+1 / 2}(i)+\Psi_{\mathrm{SH}}^{n-1 / 2}(i)\right) \\
& \left.\quad+4 \mu_{0} \varepsilon_{0} \chi^{(2)} \Delta^{2} z \Psi_{\mathrm{FU}}^{n-1 / 2}(i) \cdot\left(\Psi_{\mathrm{SH}}^{n+1 / 2}(i)-\Psi_{\mathrm{SH}}^{n-1 / 2}(i)\right)\right) \\
& \quad \Psi_{\mathrm{SH}}^{n+1}(i) \cdot\left(\mu_{0} \varepsilon_{0}\left(N^{2 \omega}\right)^{2} \Delta^{2} z\right)=\Psi_{\mathrm{SH}}^{n}(i+1) \Delta^{2} t+\Psi_{\mathrm{SH}}^{n}(i)\left(2 \mu_{0} \varepsilon_{0}\left(N^{2 \omega}\right)^{2} \Delta^{2} z-2 \Delta^{2} t\right) \\
& \quad+\Psi_{\mathrm{SH}}^{n}(i-1) \Delta^{2} t-\Psi_{\mathrm{SH}}^{n-1}(i) \mu_{0} \varepsilon_{0} n_{\mathrm{s}}^{2} \Delta^{2} z-\Psi_{\mathrm{FU}}^{n}(i) \Psi_{\mathrm{FU}}^{n+1}(i) 2 \mu_{0} \varepsilon_{0} \chi^{(2)} \Delta^{2} z \\
& \quad+\Psi_{\mathrm{FU}}^{n}(i) \Psi_{\mathrm{FU}}^{n}(i) 4 \mu_{0} \varepsilon_{0} \chi^{(2)} \Delta^{2} z-\Psi_{\mathrm{FU}}^{n}(i) \Psi_{\mathrm{FU}}^{n-1}(i) 2 \mu_{0} \varepsilon_{0} \chi^{(2)} \Delta^{2} z \\
& \quad-\Psi_{\mathrm{FU}}^{n+1 / 2}(i) \Psi_{\mathrm{FU}}^{n+1 / 2}(i) 2 \mu_{0} \varepsilon_{0} \chi^{(2)} \Delta^{2} z+\Psi_{\mathrm{FU}}^{n+1 / 2}(i) \Psi_{\mathrm{FU}}^{n-1 / 2}(i) 2 \mu_{0} \varepsilon_{0} \chi^{(2)} \Delta^{2} z \\
& \quad+\Psi_{\mathrm{FU}}^{n-1 / 2}(i) \Psi_{\mathrm{FU}}^{n+1 / 2}(i) 2 \mu_{0} \varepsilon_{0} \chi^{(2)} \Delta^{2} z-\Psi_{\mathrm{FU}}^{n-1 / 2}(i) \Psi_{\mathrm{FU}}^{n-1 / 2}(i) 2 \mu_{0} \varepsilon_{0} \chi^{(2)} \Delta^{2} z
\end{aligned}
$$

where the index $n$ represents the time evolution steps, the index $i$ is the position along the $z$-direction, $\Delta t$ is the time step, and $\Delta z$ is dimension of spatial cell. The simulations are performed by considering as source the following sinusoidal signal:

$$
\Psi_{0}^{\mathrm{FU}}=\sin \left(\omega_{0}^{\mathrm{FU}} \cdot t \cdot \Delta t\right) .
$$

As boundary conditions, the absorbing boundary conditions (ABCs) [14] are considered.

\section{QPM ridge design: Analytical and numerical results}

Usually high intensity sources are necessary in order to observe an $\mathrm{SH}$ in a $\chi^{(2)}$ nonlinear structure. For this purpose a nonlinear waveguide with quasi-phase-matched dielectric step discontinuities can increase the SH efficiency conversion. The QPM technique reduces the phase mismatch between the fundamental and the SH fields by the introduction of a grating in the waveguide in order to compensate the difference of the propagation refractive indexes $\Delta N=N^{2 \omega}-N^{\omega}$. It is useful to define the coherence length [4-6]:

$$
L c=\frac{\pi}{\left|2 \beta^{\mathrm{FU}}-\beta^{\mathrm{SH}}\right|}=\frac{\lambda_{\mathrm{FU}}}{4\left|N^{\omega}-N^{2 \omega}\right|}
$$

where $\beta^{\mathrm{FU}}$ and $\beta^{\mathrm{SH}}$ are the propagation constants of the fundamental and the $\mathrm{SH}$, respectively; $\lambda_{\mathrm{FU}}$ is the free-space wavelength of the fundamental wave; and $N^{\omega}$ and $N^{2 \omega}$ are the effective indices at 


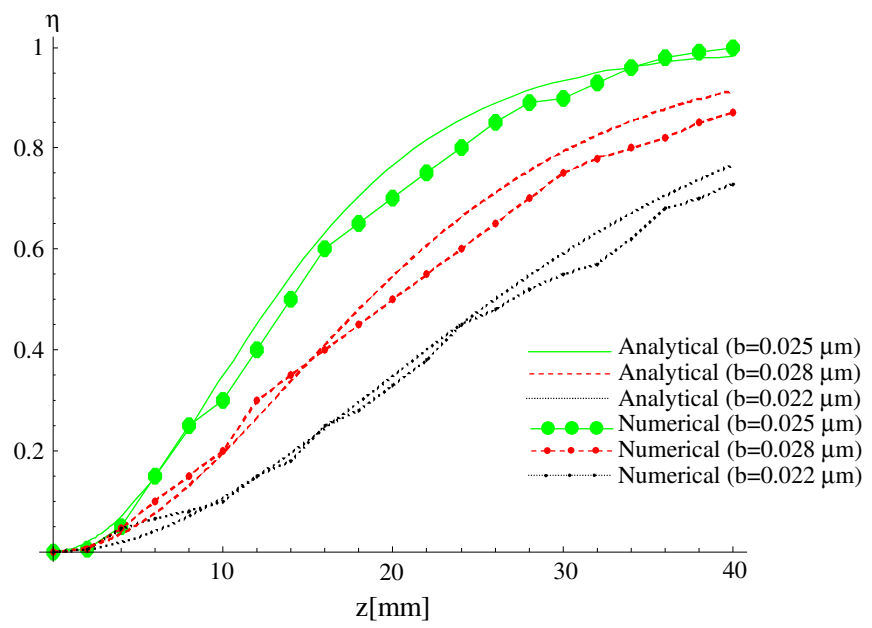

Fig. 3. Analytical and numerical conversion efficiency $\eta$ versus $z$ for different values of $b$. The plots are obtained by an input power of $P_{0}^{\omega}=100 \mathrm{~mW}$, and by considering a continuous wave as source.

\section{Table 2}

Geometrical and optical parameters of the designed ridge waveguide.

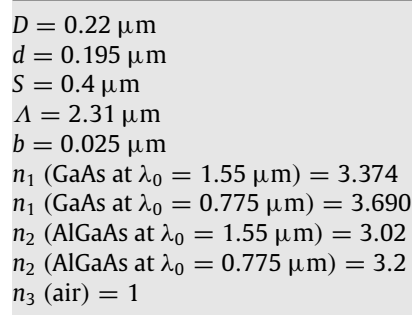

the fundamental and second-harmonic frequencies, respectively. $L c$ represents the length when the fundamental and harmonic get out of phase by $\pi$ radians with a corresponding mismatch in wave- $k$ vectors of $\Delta k=k^{\mathrm{SH}}-2 k^{\mathrm{FU}}=2 k_{0} \Delta N$, where $k_{0}=2 \pi / \lambda_{\mathrm{FU}}, k^{\mathrm{SH}}$ and $k^{\mathrm{FU}}$ are the wavevectors for the $\mathrm{SH}$ and fundamental waves, respectively. The QPM condition, applied to the grating period, is given by the relationship:

$$
\frac{2 \pi}{\Lambda}=\Delta k
$$

which is equivalent to $\Lambda=2$ Lc. This method was applied to the ridge waveguide of Fig. 1(a). The ridge waveguide facilitates the field confinement and, consecutively, the SH conversion. The thickness of this waveguide satisfies the $T E^{y}$ single mode condition for working wavelengths of $\lambda_{0}=1.55 \mu \mathrm{m}$ and $\lambda_{0}=0.775 \mu \mathrm{m}$. We calculate numerically the SH efficiency of the GaAs ridge waveguide by considering a $T E^{y}$ polarized pump source at a wavelength of $\lambda_{\mathrm{FU}}=1.55 \mu \mathrm{m}$ (SH at $\lambda_{\mathrm{SH}}=0.775 \mu \mathrm{m}$ ). In this case the simulated structure is characterized by a QPM grating with $\Lambda=2.31 \mu \mathrm{m}, S=0.4 \mu \mathrm{m}, D=0.22 \mu \mathrm{m}, d=0.195 \mu \mathrm{m}(L c=L s=\Lambda / 2), \chi^{(2)}(\mathrm{GaAs})=$ $200 \mathrm{pm} / \mathrm{V}$ [7], $n_{1}\left(\mathrm{GaAs}\right.$ at $\left.\lambda_{0}=1.55 \mu \mathrm{m}\right)=3.374, n_{1}\left(\mathrm{GaAs}\right.$ at $\left.\lambda_{0}=0.775 \mu \mathrm{m}\right)=3.690$ [15], $n_{2}\left(\right.$ AlGaAs at $\left.\lambda_{0}=1.55 \mu \mathrm{m}\right)=3.02, n_{2}\left(\mathrm{AlGaAs}\right.$ at $\left.\lambda_{0}=0.775 \mu \mathrm{m}\right)=3.2$ [16]. The SH conversion efficiency $\eta$ of Fig. 3 is calculated analytically and numerically along the waveguide as

$$
\eta=\left|A^{2 \omega}(z)\right|^{2} /\left|A^{\omega}(z=0)\right|^{2}=\left|\Psi_{\mathrm{SH}}(z)\right|^{2} /\left|\Psi_{\mathrm{FU}}(z=0)\right|^{2}
$$




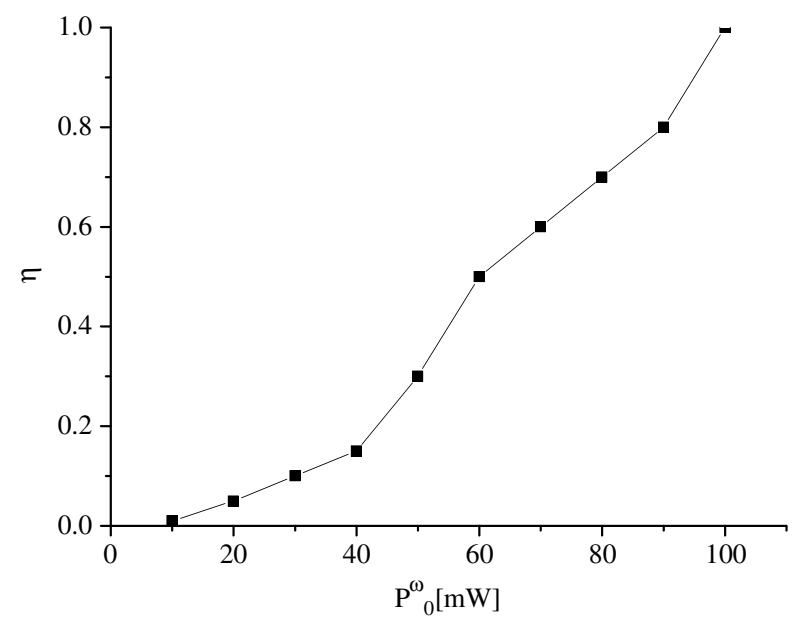

Fig. 4. Relationship between the input power $P_{0}^{\omega}$ and the SH conversion efficiency $\eta$ for a grating length of $40 \mathrm{~mm}$ by using the parameters reported in Table 2.

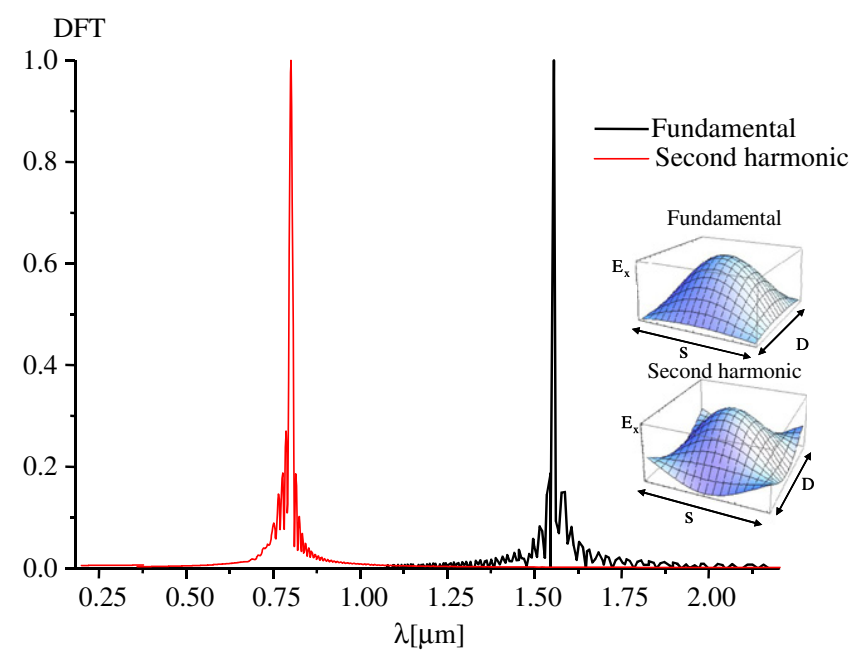

Fig. 5. DFT numerical spectra of the fundamental and SH modes at the ridge waveguide output by using a continuous wave as source. Inset: fundamental and SH mode profiles in the region II of Fig. 2.

where $A^{\omega}$ and $A^{2 \omega}$ are solutions of (1). Fig. 3 shows that for a fixed input fundamental power of $P_{0}^{\omega}=$ $100 \mathrm{~mW}$ (maximum power used for a similar GaAs/AlGaAs nonlinear waveguides reported in [3]) the best $\mathrm{SH}$ conversion efficiency is obtained by fixing the grating parameter $b$ of Fig. 1(c) to $b=0.025 \mu \mathrm{m}$ for a grating length of $40 \mathrm{~mm}$. We observe that the optimized value of $b=0.025 \mu \mathrm{m}$ takes into account the losses of the backscattered signal. A high value of $b$ provides a better SH efficiency but increases the reflectivity at each step discontinuity; for this purpose for $b=0.028 \mu \mathrm{m}$ the SH efficiency is lower than the case of $b=0.025 \mu \mathrm{m}$, but is larger if compared with the case of $b=0.022 \mu \mathrm{m}$ (see Fig. 3). The optical and the geometrical parameters of the optimized ridge waveguide are reported in Table II. A good agreement between numerical and analytical results is observed in Fig. 3, thus confirming the accuracy of the model which includes the losses due to the backscattering. As shown in Fig. 4, for a grating length of $40 \mathrm{~mm}$, the SH conversion efficiency decreases by reducing the input power. The final spectra at the ridge waveguide output and the analytical modal profiles of the fundamental and $\mathrm{SH}$ modes used during the calculus are reported in Fig. 5. The numerical spectra are obtained through 


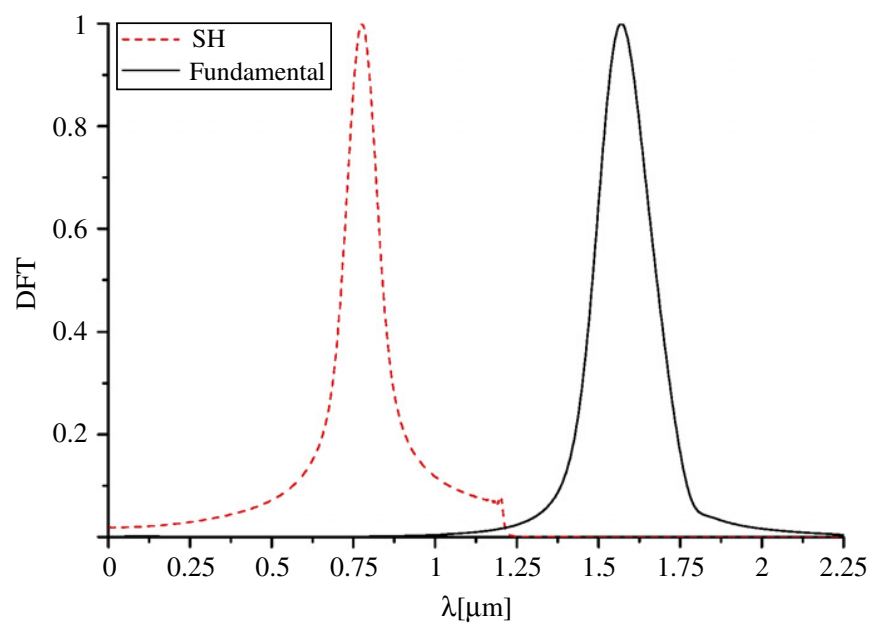

Fig. 6. DFT numerical spectra of the fundamental and SH modes at the ridge waveguide output by using a carrier modulated by an exponential signal.
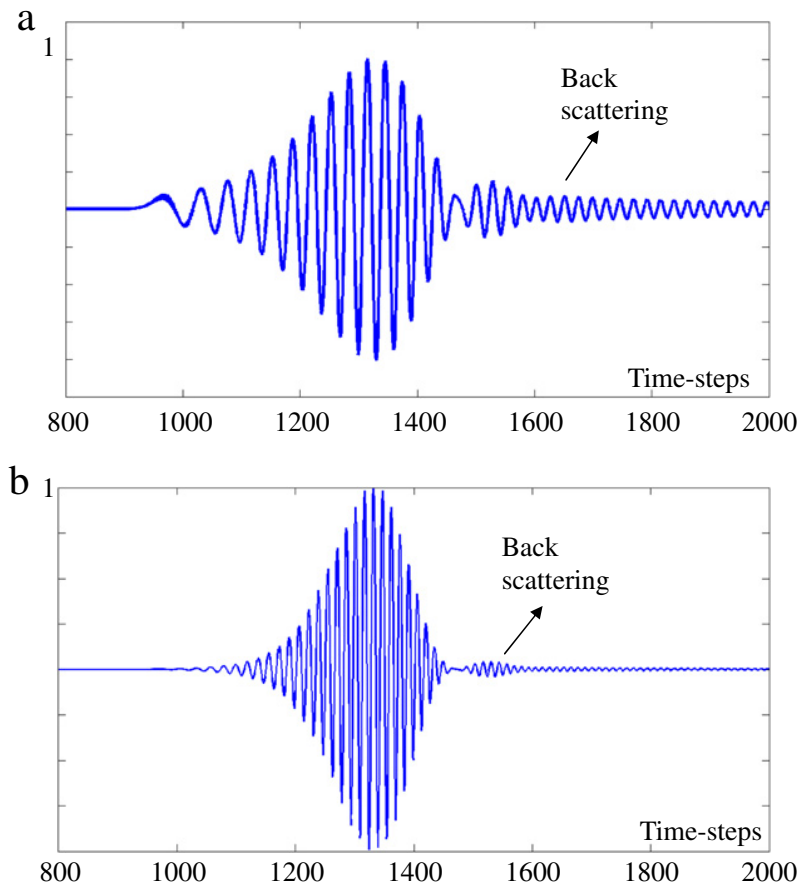

Fig. 7. Time evolution of the fundamental (a) and SH (b) mode in a reference point located after some grating periods.

the Discrete Fourier Transform (DFT) of $\psi_{\mathrm{FU}}$ and $\psi_{\mathrm{SH}}$ by considering as source the continuous wave reported in Eq. (13). Regarding filtering application an important aspect of the design is the bandwidth of the signal at the ridge waveguide output: in order to obtain a larger bandwidth we consider a carrier modulated by an exponential signal defined by

$$
\Psi_{0}^{\mathrm{FU}}=\exp \left(-\left(t \cdot \Delta t / T_{0}\right)^{2}\right) \sin \left(\omega_{0}^{\mathrm{FU}} \cdot t \cdot \Delta t\right)
$$



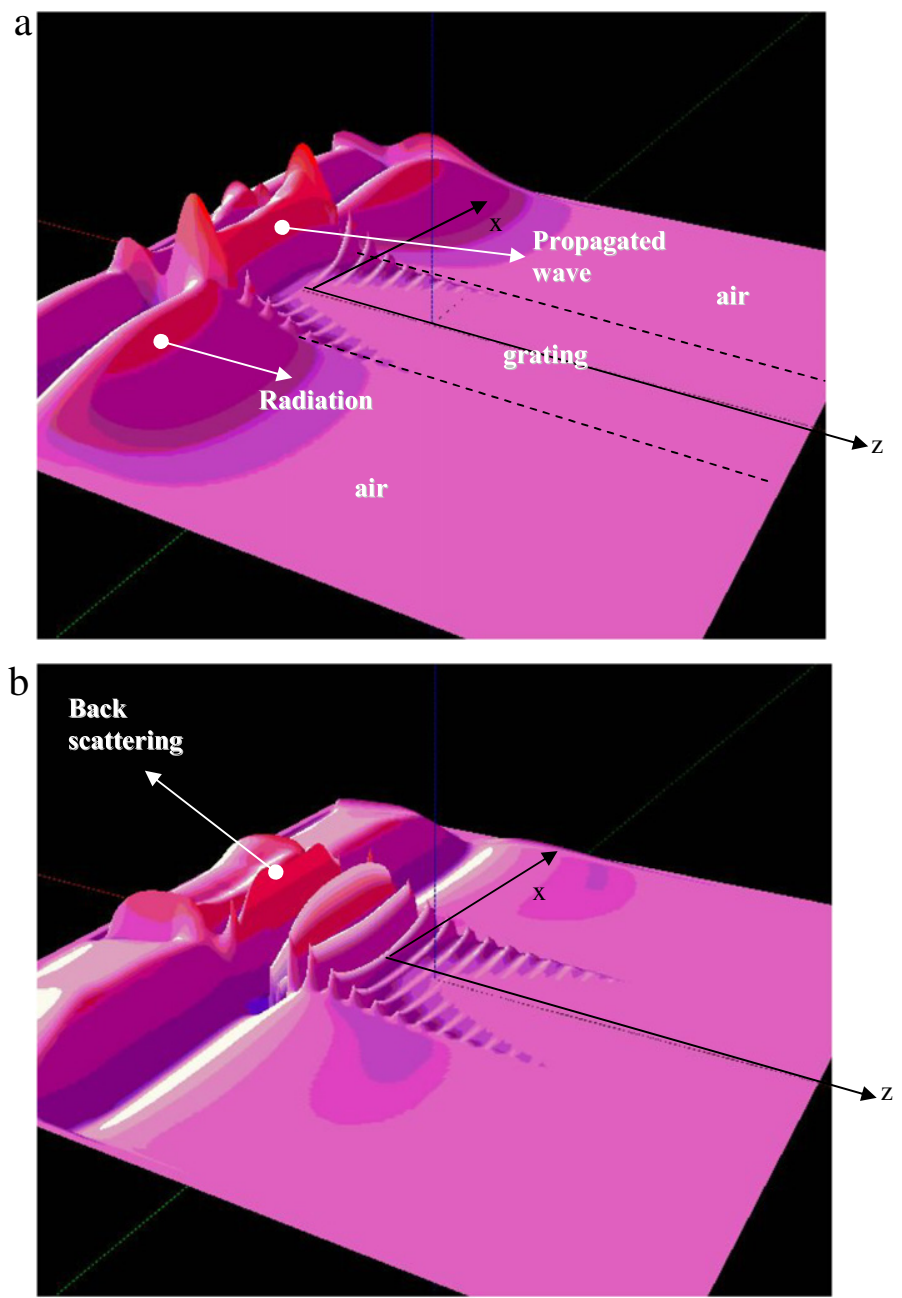

Fig. 8. Fundamental 2D field evaluated on the grating region at two different time steps: (a) propagated wave and radiated field at time step $t_{1}$, (b) propagated and backscattered wave at time step $t_{2}>t_{1}$.

In this case the spectra evaluated at the output are reported in Fig. 6, By using as parameters $T_{0}=300 \times 10^{-16} \mathrm{~s}, \Delta t=1.6679 \times 10^{-16} \mathrm{~s}$ (time step), and $\Delta z=0.005 \times 10^{-6} \mu \mathrm{m}$ (spatial step size) we obtain approximately the same order of the efficiency reported in Fig. 3 . The modulated Gaussian pulse is also used in order to analyze better the backscattering generated by grating. Figs. 7 and 8 show the generated backscattered waves at different time steps in a point located after some grating periods and in the 2D ridge periodic plane, respectively: in both the cases the backscattered wave is observed. Moreover Fig. 8 shows also the signal radiated in the air region (radiation losses).

We summarize the design criteria of the ridge waveguide in the following steps:

(i) In order to minimize the group velocity dispersion and the walk-off effect we design the GaAs/AlGaAs waveguide ( $T E^{y}$ excitation hypothesis) with a single fundamental and $\mathrm{SH}^{T} E^{y}$ copropagating mode by fixing the parameter $S, D$ and $d$;

(ii) we design the periodicity $\Lambda$ of the grating through the QPM condition;

(iii) we optimize the grating by analyzing the key parameter $b$ by balancing the losses due to the backscattering with respect to the enhancement of the etched profile. 


\section{Conclusion}

In this work we optimize a 3D GaAs/AlGaAs ridge second harmonic converter by using a rigorous analysis of the fundamental/SH coupled equations which provides the conversion efficiency of the QPM grating versus the device length. The accuracy of the design approach is proved by a good agreement between analytical and numerical results. The model can be extended also to complex 3D nonlinear waveguides with defects.

\section{References}

[1] S.J. Wagner, B.M. Holmes, U. Younis, A.S. Helmy, J.S. Aitchison, D.C. Hutchings, Appl. Phys. Lett. 94 (2009) 151107.

[2] X. Yu, L. Scaccabarozzi, A.C. Lin, M.M. Fejer, J.S. Harris, J. Cryst. Growth 301 (2007) 163.

[3] M.H. Chou, I. Brencr, K.R. Parameswaran, M.M. Fejer, Electron. Lett. 35 (1999) 978.

[4] E.U. Rafailov, P.L. Alvarez, C.T.A. Brown, W. Sibbett, R.M. De la Rue, P. Millar, D.A. Yanson, J.S. Roberts, P.A. Houston, Opt. Lett. 26 (2001) 1984.

[5] T. Suhara, M. Fujimura, Waveguide Nonlinear-Optic Devices, Springer, Berlin, 2003.

[6] T. Suhara, H. Nishihara, IEEE J. Quantum Electron. 8 (1972) 661.

[7] A. Massaro, V. Tasco, M.T. Todaro, R. Cingolani, M. De Vittorio, A. Passaseo, Opt. Express 16 (2008) 14496.

[8] T. Rozzi, M. Farina, Advanced electromagnetic analysis of passive and active planar structures, in: IEE Electromagnetic Wave Series, vol. 46, London, 1999.

[9] C.G. Someda, Onde elettromagnetiche, UTET Ed., Torino, 1996.

[10] M.A. Alsunaidi, H.M. Masoudi, J.M. Arnold, IEEE Photonics Technol. Lett. 12 (2000) 395.

[11] A. Massaro, T. Rozzi, Opt. Express 14 (2006) 2027.

[12] T. Rozzi, M. Mongiardo, Open Electromagnetic Waveguides, in: IEE Electromagnetic Waves Series, vol. 43, London, 1997.

[13] A. Taflove, S.C. Hagness, Computational Electrodynamic: The Finite-difference Time-Domain Method, 2nd ed., Arthec House Publishers, London, 2000.

[14] G. Mur, IEEE Trans. Electromagn. Compat. 23 (1981) 3.

[15] J.S. Blakemore, J. Appl. Phys. 53 (1982) 123.

[16] A.N. Pikhtin, A.D. Yas'kov, Sov. Phys. Semicond. 14 (1980) 389. 